\title{
Improving the Design of Industrial Microwave Processing Systems through Prediction of the Dielectric Properties of Complex Multi-layered Materials
}

Juliano Katrib*, Olaosebikan Folorunso, Christopher Dodds, Georgios Dimitrakis and Sam W Kingman

National Centre for Industrial Microwave Processing (NCIMP), Energy and Sustainability Research Division, Faculty of Engineering, University of Nottingham, Nottingham, NG7 2RD, United Kingdom

juliano.katrib@nottingham.ac.uk, enxof5@nottingham.ac.uk, chris.dodds@nottingham.ac.uk, georgios.dimitrakis@nottingham.ac.uk, sam.kingman@ nottingham.ac.uk

*Corresponding author: juliano.katrib@ nottingham.ac.uk, telephone number: 00441159514104

Keywords: Dielectric Property, Clausius-Mossotti, Mixture Equations, Vermiculite, Microwave Processing

\begin{abstract}
Rigorous design of industrial microwave processing systems requires in-depth knowledge of the dielectric properties of the materials to be processed. These values are not easy to measure, particularly when a material is multi-layered containing multiple phases, when one phase has a much higher loss than the other and the application is based on selective heating. This paper demonstrates the ability of the Clausius-Mossotti (CM) model to predict the dielectric constant of multi-layered materials. Furthermore, mixing rules and graphical extrapolation techniques were used to further evidence our conclusions and to estimate the loss factor. The material used for this study was vermiculite, a layered alumina-silicate mineral containing up to $10 \%$ of an interlayer hydrated phase. It was measured at different bulk densities at two distinct microwave frequencies, namely 934 and $2143 \mathrm{MHz}$. The CM model, based on the ionic polarisability of the bulk material, gives only a prediction of the dielectric constant for experimental data with a deviation of less than $5 \%$ at microwave frequencies. The complex refractive index model (CRIM), Landau, Lifshitz and Loyenga (LLL), Goldschmidt, Böttcher and Bruggeman-Hanai model equations are then shown to give a strong estimation of both dielectric constant and loss factor of the solid material compared to that of the measured powder with a deviation of less than $1 \%$. Results obtained from this work provide a basis for the design of further electromagnetic processing systems for multi-layered materials consisting of both high loss and low loss components.
\end{abstract}

Keywords: Clausius-Mossotti, Mixture Equations, Dielectric Property, Vermiculite

\section{Introduction}

The world is under increasing pressure to develop new, more energy efficient technology that enables the production of less waste and is ultimately more sustainable [1]. Material processing through microwave 
energy has received attention in the past few years for delivering benefits in a number of different applications including; food, fine and bulk chemicals, oil, gas, minerals and metal extraction [2]. Despite the potential to deliver a step change in overall process efficiency across a diverse range of sectors, the benefits of microwave technology are not being realised.

The dielectric properties characterisation of multi-layered materials has so far been empirical, because no exact theory is currently known which relates the macroscopic properties of multiphase composites to their microscopic properties [3].

The calculation of dielectric properties of multiphase materials by using the dielectric properties and physical characteristics of each of their constituent phases has received considerable attention in the past [4]. Multiphase materials are increasingly used in diverse microwave application areas, but electromagnetic analysis and characterisation efforts are severely lagging behind. The dielectric properties of a material determine how electromagnetic energy will interact with it and are expressed in the form of a complex number (Equation (1)) consisting of a real part ( $\varepsilon^{\prime}$, dielectric constant) and an imaginary part ( $\varepsilon^{\prime \prime}$, loss factor).

$$
\varepsilon^{*}=\varepsilon^{\prime}-j \varepsilon^{\prime \prime}
$$

At microwave frequencies (300 MHz-300 GHz), the dielectric constant dictates the ability of a dielectric material to be polarised (or store microwave energy within its structure through polarisation), while the loss factor expresses the ability of a material to dissipate and transform the stored electromagnetic energy into heat [5]. Given the complexities of composite systems, the concept of "effective properties" has become widely accepted [6] although from a design perspective this can lead to errors. Complex materials are multi-component and/or multiphase (state) systems. The behaviours of the material are dictated by each and every component of each individual phase, and the overall macroscopic properties are not equal to that of any single constituent, rather they are collective with weighted contribution from all components forming the system. Therefore, the effective properties are actually the equivalent properties of a hypothetical simple material (homogeneous with single component and phase) which yields the same response as that of the complex one at the same given conditions and excitations [6]. Mixture theories attempt to describe the macroscopic dielectric response of a composite material in terms of the electromagnetic properties of the constituents. Such theories are inherently approximate because they are derived by averaging an idealised model of the microscopic structure, and different mixing rules predict different results for the same mixture.

There is a significant need for designing industrial processing techniques with a sustainable form of energy in terms of carbon footprint, and increasing energy efficiency by $20 \%$ with subsequent reduction of greenhouse gases emission by $20-30 \%$ of the 1990 levels is the target of the European Union for 
sustainable growth by 2020 [1]. Therefore, there is undoubtedly demand for processes which are more efficient in terms of energy consumption, more flexible in terms of operational characteristics and ultimately, more sustainable than the current state of the art. One technology which has the potential to realise these benefits is microwave heating [7]. Its huge success in processing of food materials created an opportunity for its industrial applications in the field of mineral processing [8]. In recent years, microwave energy has also been used in the processing of wide varieties of feedstock, such as food stuffs, agricultural products, polymers, waste treatment, and microwave treatment of coal [8-10]. The concept of using microwave energy to exfoliate vermiculite is not new, but there is a lack of understanding and characterising the dielectric behaviour of this material at microwave frequencies. The accurate knowledge of the dielectric properties of vermiculite can likewise be useful in the application of electrical separation technology from separating vermiculite ores from gangue materials during the mining and concentration process with a frequency varied from $0 \mathrm{~Hz}$ to $1 \mathrm{MHz}$ [11], and is crucial for the optimum design of continuous microwave processing systems which have been shown in the literature to offer step changes in the efficiency of the exfoliation process [7].

The hypothesis presented in this paper is that a multi-layered material has different compounds interacting with each other; these compounds will affect the dielectric properties of the material and therefore the heating efficiency of the microwave process. The objectives of this work are to measure the dielectric properties of a complex multi-layered material by examining the influence of the constituent phases upon the overall dielectric response of the system and provide a theoretical background for understanding and predicting the macroscopic dielectric properties of a multi-layered material. The CM model is used to connect the real part of the relative permittivity to the polarisability of the molecules constituting the material (vermiculite). The relative permittivity is a bulk (macroscopic) property and polarisability is a microscopic property of matter; hence the relation bridges the gap between directly-observable macroscopic properties and the microscopic molecular properties. One of the limitations of the CM model is that it calculates only the dielectric constant with no explicit relationship for the loss factor. Therefore mixing rules that combine the permittivity (dielectric constant and loss factor) of each component and various structural parameters of the mixture are also used to estimate dielectric properties of the mixture and are supported by graphical extrapolation techniques. 


\section{Materials and dielectric measurement technique \\ 2.1. Sample preparation}

A vermiculite sample obtained from the Palabora mine in South Africa was used to study the correlation between the dielectric properties and bulk density of vermiculite at microwave frequencies of 934 and 2143 MHz. The chemical composition of the vermiculite sample was determined using X-ray fluorescence (XRF) with the results shown in Table 1. The total weight percentage of the identified chemical compounds (oxides) reported $(99.92 \%)$ is slightly less than the nominal $100 \%$ because the oxides of some identified elements such as $\mathrm{Be}$ and $\mathrm{Zn}$, which were less than $0.01 \%$, were omitted.

Table 1 Chemical composition of Palabora vermiculite determined using XRF

\begin{tabular}{|lllllllllllll|}
\hline & $\mathbf{S i O}_{2}$ & $\mathbf{T i O}_{2}$ & $\mathbf{A l}_{2} \mathbf{O}_{\mathbf{3}}$ & $\mathbf{M g O}$ & $\mathbf{K}_{\mathbf{2}} \mathbf{O}$ & $\mathbf{C a O}$ & $\mathbf{F e}_{2} \mathbf{O}_{3}$ & $\mathbf{N a}_{2} \mathbf{O}$ & $\mathbf{C r}_{2} \mathbf{O}_{3}$ & $\mathbf{N i O}$ & $\mathbf{M n O}$ & LOI \\
\hline Palabora(\%) & 40.8 & 1.0 & 8.6 & 21.9 & 4.8 & 5.7 & 8.5 & 0.08 & 0.050 & 0.024 & 0.07 & 8.4 \\
\hline
\end{tabular}

The XRF results shown in Table 1 were used to estimate the structural formula of the material using an approach published within the literature $[12,13]$. The obtained results show that the structural formula is similar to that determined by [14]. The oxides $\left(\mathrm{Na}_{2} \mathrm{O}, \mathrm{Cr}_{2} \mathrm{O}_{3}, \mathrm{NiO}\right.$ and $\left.\mathrm{MnO}\right)$ which were less than $0.1 \%$ by weight were neglected; it was assumed that there were 44 cation and anion charges per unit cell of clay mineral [15]. The calculated half unit cell structural formula of the vermiculite sample is given in Equation (2).

The first step taken in calculating the structural formula was the calculation of the gram-equivalents of cationic constituents in the structure, by dividing the percentage weight of each constituent present by a factor obtained from the division of the molecular weight of that constituent by the number of cationic valencies in the molecular formula. The sum of the gram-equivalents of the cationic constituents was then divided by 44 , which is the cationic and anionic valence of an idealised unit cell, to obtain the factor used for conversion of cationic valencies per unit cell. Lastly, the number of cations per unit cell was obtained by dividing the gram-equivalents of each cationic constituent by the factor calculated previously. The obtained molecular formula of vermiculite was used as an input for the calculation of the dielectric constant using the $\mathrm{CM}$ equation from the values of the polarisability of each of the constituent elements.

$\left(\mathrm{Si}_{3} \mathrm{Al}_{0.74} \mathrm{Fe}_{0.26}^{3+}\right)\left(F e_{0.21}^{3+} \mathrm{Ti}_{0.06} \mathrm{~K}_{0.45^{M g_{2.28}}}\right) \mathrm{O}_{10}(\mathrm{OH})_{2}\left(\mathrm{Ca}_{0.45^{M g_{0.12}}}\right) \mathrm{HH}_{2} \mathrm{O}$

For the dielectric measurement, riffling was used to divide bulk sample of vermiculite (about 50g) into smaller representative samples used for the dielectric characterisation. The obtained representative bulk sample was slowly pulverised by hand using an agate mortar and pestle to prevent steel contamination from milling. The sample was sieved and particles within the $-180+38 \mu \mathrm{m}$ size class were selected for measurement. The chosen range is based on the size of glass tube to obtain high packing density comparable to the processing conditions [7]. 


\subsection{Cavity Perturbation Method}

Dielectric measurements were carried out with the use of the cavity perturbation method [16]. The system used was based upon a copper resonating cylindrical cavity with the first two $\mathrm{TM}_{0 \mathrm{n} 0}$ identified modes; $\mathrm{TM}_{010}$ at $934 \mathrm{MHz}$ and $\mathrm{TM}_{020}$ at $2143 \mathrm{MHz}[17,18]$. The cavity perturbation method was chosen as it is known to be better suited to measurements of low loss materials and can be used with pulverised samples [19]. As the requirement for the specimen size is very small, the sample is easily prepared and no calibration is required as the measurement is based on the differential effects of an empty and loaded cavity. The measurement error for $\varepsilon^{\prime}$ is less than $2 \%$ [20]. In addition, the vermiculite sample available for measurement was in granular form and unlike transmission/reflection techniques there is a lack of resolution for low loss materials (where loss is less than 0.05 ).

The experimental setup used here is detailed in previous work [21]. The system consists of a resonant cylindrical cavity (internal diameter $190 \mathrm{~mm}$ and internal height $19 \mathrm{~mm}$ ) with 2 ports. The vector network analyser (Hewlett Packard 8753ES) measures the transmitted signal between the 2 ports. The resonant system is based on the well-established system developed by Hutcheon where extensive numerical modelling was performed [22] to establish the uncertainty and the accuracy of the system. The study claims that the error of the technique was shown to be less than $\pm 3 \%$ for $\varepsilon_{\mathrm{r}} \leq 30$ and another study claimed that the relative percentage error was less than 1\% for PTFE rod [21]. Care was taken to ensure the sample was placed in the region of maximum electric field in the cavity, and the resonant frequency $\left(f_{1}\right)$ and quality factor $\left(Q_{1}\right)$ of the sample were measured. Each measurement was repeated fifteen times at the different selected bulk densities and the mean value of measurements the associated standard deviation and the relative standard errors were computed [23].

\section{Determination of the Dielectric properties of a heterogeneous mixture}

Mixing rules and graphical extrapolation techniques have been used to estimate the dielectric properties of vermiculite at its solid density from that of the pulverised sample. Figure 1 illustrates the transformation of microscopic polarisation of different constituents in a mixture to give effective macroscopic polarisation of the bulk mixture. 


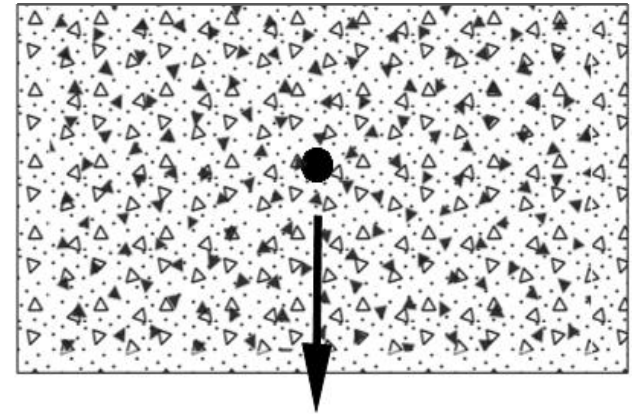

Air - particle mixture

$$
P=\sum_{i=1}^{n} \bar{P}_{i} \nu_{i}
$$

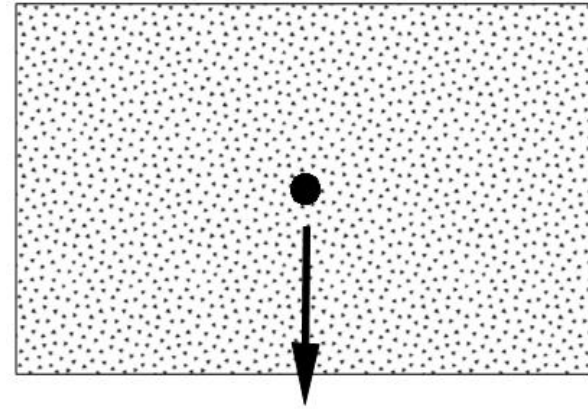

Particle - natural density

Fig. 1 Illustration of transformation of polarisation from microscopic scale to macroscopic scale, the macroscopic polarisation, $\mathrm{P}\left(\mathrm{C} / \mathrm{m}^{2}\right)$ is equal to the average for all microscopic polarisations $\overline{\boldsymbol{P}}\left(\mathrm{C} / \mathrm{m}^{2}\right)$, and $v_{i}$ is the fractional volume of each of the constituents [24]

Where $\mathrm{P}\left(\mathrm{C} / \mathrm{m}^{2}\right)$ is effective polarisation, $\bar{P}_{i}\left(\mathrm{C} / \mathrm{m}^{2}\right)$ is the polarisation contributed by each of the constituents in the mixture and $v_{i}$ is the fractional volume of each of the constituents. When a multiphase system such as vermiculite is subjected to an electric field, the individual phases in the mixture will maintain their unique electromagnetic properties and will, therefore, be polarised differently within the bulk mixture. The effective polarisation of the mixture to the electric field is the summation of the individual polarisation of its constituents. The effective polarisation obtained on the right hand side of Figure 1 is the summation of the individual polarisations of each of the constituents present in the mixture. In a complex multiphase system such as vermiculite, dielectric mixing equations can be used to estimate the bulk dielectric properties of the material from the summation of the dielectric properties of the different mineral phases present as a function of the their fractional volume.

\subsection{Calculation of dielectric properties using the Clausius-Mossotti and mixture equations models}

The CM model (Equation (3)) which relates dielectric constant to dielectric polarisability was used to quantify the dielectric constant of vermiculite from its calculated mineral dielectric polarisability. The accuracy of the CM model depends on how closely the real microstructure of the sample corresponds to the idealised microscopic model used in deriving the specific mixture relation [25].

$\left.\varepsilon^{\prime}=\frac{\left(3 V_{m}+8 \pi \alpha \alpha_{D}\right)}{\left(3 V_{m}-4 \pi \alpha \alpha_{D}\right.}\right)$

Where $V_{m}$ is the molecular volume $\left(\AA^{3}\right)$ of vermiculite and $\alpha_{D}\left(\AA^{3}\right)$ is the mineral polarisability [26,27].

The additivity rule was used to obtain the mineral dielectric polarisability [26] of the vermiculite from the summation of the atomic or ion polarisability of all of the elements present in the structural formula shown in Equation (2). For example in Equation (4), the mineral polarisability of substance $\mathrm{X}_{2} \mathrm{Y}_{3} \mathrm{Z}_{4}$ given 
on the left hand side is calculated on the right hand side from the individual ionic polarisability of its elemental components.

$$
\left(X_{2} Y_{3} Z_{4}\right)=2 \alpha_{X}\left(X^{2+}\right)+3 \alpha_{Y}\left(Y^{3+}\right)+4 \alpha_{Z}\left(Z^{4+}\right)
$$

The data of the ionic polarisabilities used in this work was obtained from the literature $[26,27]$ and the vermiculite molecular volume $\left(\mathrm{V}_{\mathrm{m}}\right)$ was calculated from Equation (5).

$$
V_{m}=\frac{\text { Molecular mass of vermiculite }}{\text { Particle density }\left(\mathrm{g} / \mathrm{cm}^{3}\right) * \text { Avogrados number }}
$$

The calculated dielectric polarisability and molecular volume of vermiculite obtained from Equations (4-5) are $299.91 \AA^{3}$ and $44.49 \AA^{3}$ respectively. These values were entered into Equation (3) to obtain 5.92 as the estimated dielectric constant of vermiculite. The estimated dielectric constant obtained from this technique can be used as representative of that of solid vermiculite at a solid density of $2.71 \mathrm{~g} / \mathrm{cm}^{3}$ and also as a reference value for comparison with the values obtained from mixing rules and graphical extrapolation.

Furthermore, mixture equations attempt to describe the macroscopic dielectric response of a composite material in terms of the electromagnetic properties of the constituents. Several quantitative mixture equations have been proposed and used for the estimation of the dielectric properties of both homogenous and heterogeneous materials [24,28-31].

Extensive literature searches have revealed no published work which has applied mixture equations to multi-layered materials such as vermiculite. Table 2 presents the mixing rule equations used in this work. A critical review of the fundamental theories, derivations and theoretical assumptions of these rules can be found in the literature [30-33]. 
Table 2 Dielectric mixture equations

\begin{tabular}{|c|c|}
\hline$\varepsilon_{m}^{1 / 2}=v_{a} \varepsilon_{a}^{1 / 2}+v_{s} \varepsilon_{s}^{1 / 2}$ & $\begin{array}{l}\text { Complex refractive index model } \\
\text { (CRIM) }\end{array}$ \\
\hline$\varepsilon_{m}^{1 / 3}=v_{a} \varepsilon_{a}^{1 / 3}+v_{s} \varepsilon_{s}^{1 / 3}$ & $\begin{array}{l}\text { Landau, Lifshitz and Loyenga model } \\
\text { (LLL) }\end{array}$ \\
\hline$\frac{\varepsilon_{m}-\varepsilon_{a}}{3 \varepsilon_{m}}=v_{s} \frac{\varepsilon_{s}-\varepsilon_{a}}{\varepsilon_{s}+2 \varepsilon_{m}}$ & Böttcher model \\
\hline$\varepsilon_{m}=\frac{\varepsilon_{a}\left\{\varepsilon_{a}+\left(\varepsilon_{s}-\varepsilon_{a}\right)\left(v_{s}+f v_{a}\right)\right\}}{\varepsilon_{a}+f v_{a}\left(\varepsilon_{s}-\varepsilon_{a}\right)} 0 \leq f \leq 1 \quad f=1 / 9$ & Goldschmidt model \\
\hline$\frac{\varepsilon_{m}-\varepsilon_{s}}{\varepsilon_{a}-\varepsilon_{s}}\left(\frac{\varepsilon_{a}}{\varepsilon_{m}}\right)^{\alpha}=1-v_{a} ; \alpha=1 / 3$ & Bruggeman-Hanai model \\
\hline \multicolumn{2}{|c|}{$\begin{array}{l}\varepsilon_{\mathrm{m}} \text { and } \varepsilon_{\mathrm{i}} \text { permittivity of the measured mixture and its constituents respectively } \\
\alpha \text { is a depolarisation factor, it ranges from -1 to } 1 \\
\text { " } f \text { " relates the orientation of the molecular structure of materials to the direction of the applied electric field } \\
\varepsilon_{a} \text { represents the permittivity of air, } \varepsilon_{s} \text { is the permittivity of the solid particle and } \varepsilon_{m} \text { is the permittivity of the "air-particle" mixture } \\
v_{a} \text { and } v_{s} \text { are the volume fractions of the air and solid particles respectively }\left(\mathrm{v}_{\mathrm{a}}+\mathrm{v}_{\mathrm{s}}=1\right)\end{array}$} \\
\hline
\end{tabular}

The particle density of vermiculite has been measured using helium gas pycnometry (AccuPyc ${ }^{\mathrm{TM}} 1330$ Pycnometer), the measured density is $2.71 \mathrm{~g} / \mathrm{cm}^{3}$ and is comparable to values in the literature [34]. The complex permittivity of air $\left(\varepsilon_{\mathrm{a}}\right)$ is given as $1.000649-j 0$, where the dielectric constant is 1.000649 and loss factor is 0 [23].

Graphical extrapolation techniques based on the density dependence of the dielectric properties of granular or pulverised materials has been previously used for predicting the dielectric properties of bulk solid samples of coal, limestone, wheat flour and fish [4,35-37]. In this work, graphical extrapolation was proposed to support the mixing equations and has been applied for estimating the dielectric properties of solid vermiculite. Kent expressed the permittivity of a fish meal sample as a quadratic function of density as expressed in Equations (6) and (7). Where $\partial, \beta, \alpha$ and $\delta$ are constants peculiar to the measured material [35].

$\varepsilon^{\prime}=\partial \rho^{2}+\beta \rho+1$

$\varepsilon^{\prime \prime}=\alpha \rho^{2}+\delta \rho+0$

Klein reported a linear relationship between $\sqrt{\varepsilon^{\prime}}$ and the bulk density of coal shown in Equation (8), where " $\mathrm{m}$ " is the slope of the graph. Comparisons of second order polynomial function (Equation (6)) and 
the linear relationship between $\sqrt{\varepsilon^{\prime}}$ and bulk density described in Equation (8) show that the constants $\partial$

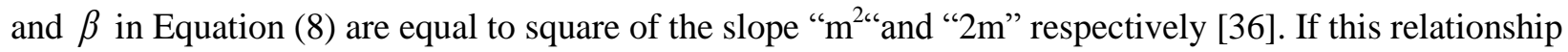
holds for any material, measurement of the dielectric constant at any specific and known bulk density can be used to determine the dielectric constant at any density up to the natural density of its solid material. Nelson showed a linear relationship between the loss factor and density by completing the square of Equation (7) to obtain Equation (9) [4]. This can be extrapolated to determine the loss factor of a solid particle of any material.

$$
\begin{aligned}
& \sqrt{\varepsilon^{\prime}}=m \rho+1 \\
& \sqrt{\varepsilon^{\prime \prime}+\frac{\delta^{2}}{4 \alpha}}=\sqrt{\alpha} \rho+\sqrt{\frac{\delta^{2}}{4 \alpha}}
\end{aligned}
$$

\section{Results and Discussion}

This work describes the macroscopic dielectric response of multi-layered material versus bulk densities at room temperature $\left(20^{\circ} \mathrm{C} \pm 1{ }^{\circ} \mathrm{C}\right)$. The permittivity of pulverised Palabora vermiculite was measured across a range of bulk densities $\left(0.84-1.42 \mathrm{~g} / \mathrm{cm}^{3}\right)$ at 934 and $2143 \mathrm{MHz}$ at room temperature using the cavity perturbation technique previously discussed in section 2.2 . The approach was based on testing the validity of the CM theory to estimate the dielectric constant of a multi-layered material by using the properties of constituent phases and the molecular formula of vermiculite. Many studies have reported that CM shows some weakness with dipolar materials and the calculation of the local field is quite complicated, however, the results demonstrate controversial behaviour compared to the theory and therefore, the need to validate the data by different approach. Mixing rules and the graphical extrapolation technique were considered to be the best candidate for this purpose.

Figure 2 shows the relationship between the measured complex permittivity of pulverised vermiculite and bulk density. The average dielectric constant and loss factor increase from 2.13-3.18 and 0.09-0.20 with an increase in density from 0.849 to $1.42 \mathrm{~g} / \mathrm{cm}^{3}$. The range of the standard deviation for the dielectric constant is between \pm 0.00630 and \pm 0.01498 at both frequencies and the range of standard deviation on the loss factor is between \pm 0.00262 and \pm 0.00158 for both frequencies respectively. The average dielectric constant at $934 \mathrm{MHz}$ was shown to be slightly higher than at $2143 \mathrm{MHz}$ at all selected bulk densities, however the reverse is the case for loss factor as higher values were recorded at $2143 \mathrm{MHz}$. The higher dielectric constant at $934 \mathrm{MHz}$ is due to the strong presence of dipolar polarisation which produces higher net polarisation effects at $934 \mathrm{MHz}$ [38]. The higher loss factor recorded at $2143 \mathrm{MHz}$ is, likely to be due to the increase in the rate of polarisation of the free water in the vermiculite structure. 


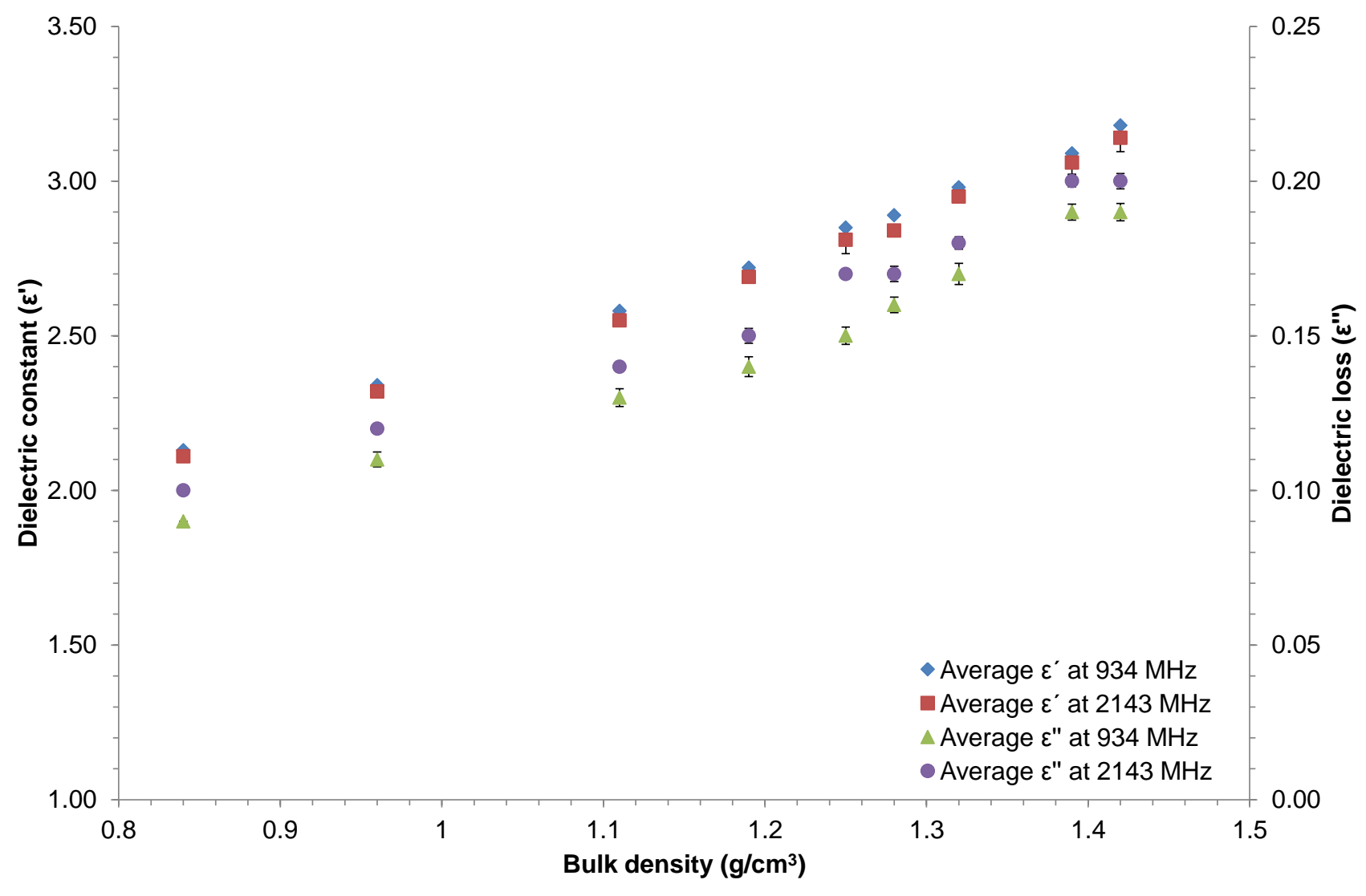

Fig. 2 Average dielectric properties of vermiculite as a function of bulk density at frequencies of $934 \mathrm{MHz}$ and 2143 MHz. The blue diamond represents the average dielectric constant at $934 \mathrm{MHz}$ and the red square denotes the average dielectric constant at $2143 \mathrm{MHz}$. The green triangle denotes the average loss factor at $934 \mathrm{MHz}$ and the purple circle denotes the average loss factor at $2143 \mathrm{MHz}$. Error bars are plotted plus or minus one standard deviations

Figure 3 and Figure 4 show the second order polynomial curves for the dielectric constant and loss factor for pulverised vermiculite. This is the best fit obtained from the plotted graph with a coefficient of regression higher than 0.99 for both measurements at the two measured frequencies. Second order polynomial curves have the highest coefficient of regression for all the curve fitting methods tried and this agrees with the previous results published in the literature for different materials [4]. This finding is a result of both the permittivity and the loss factor being quadratic functions of the density of the granular material [39]. The regression equations obtained for both dielectric constant and loss factor are also consistent with Equations (8-9). 


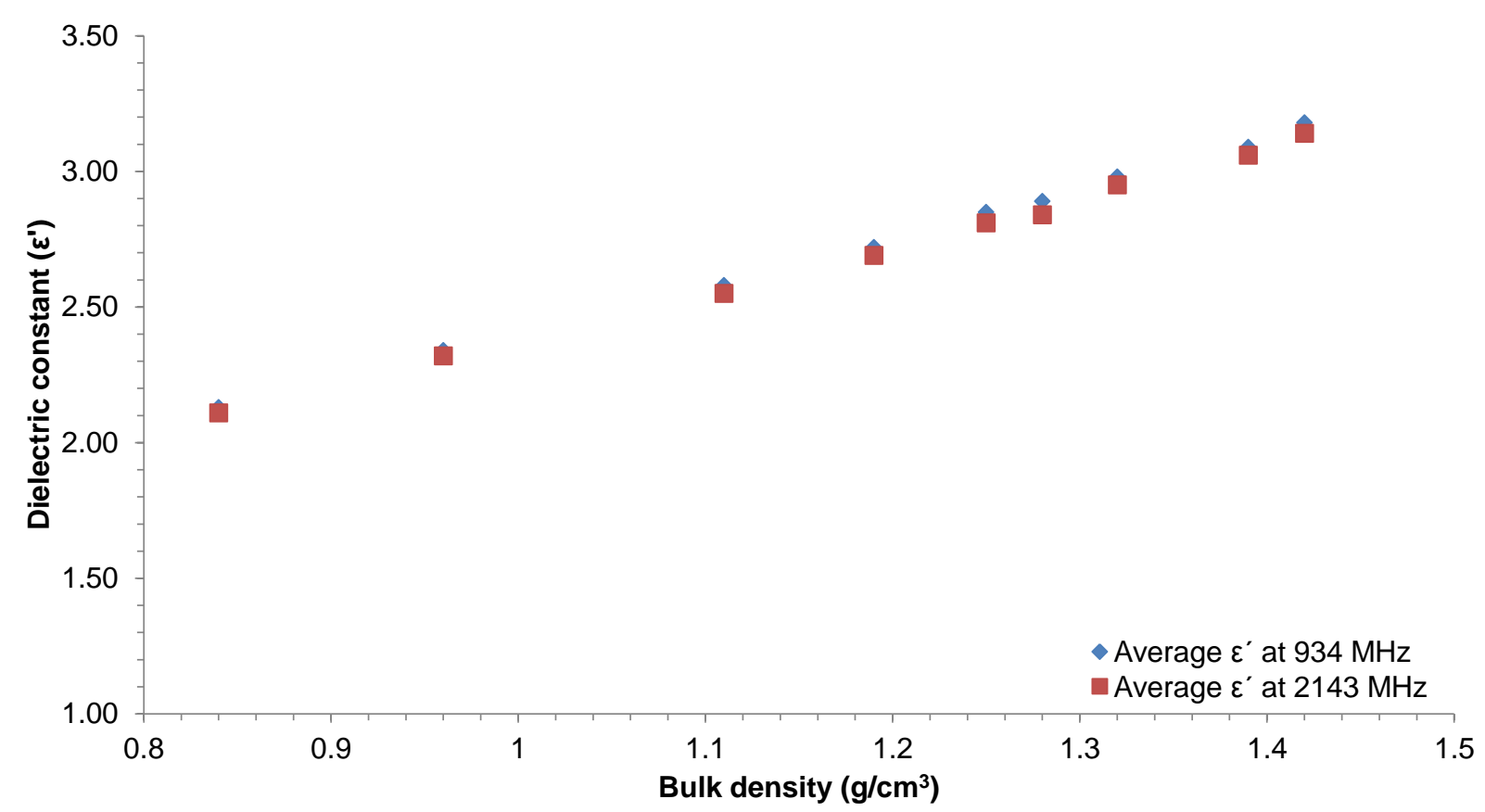

Fig. 3 Second order polynomial regression curve of the dielectric constant at 934 and $2143 \mathrm{MHz}$ of pulverised vermiculite against bulk density. The blue diamond presents the average calculated dielectric constant at $934 \mathrm{MHz}$ and the red square represents average dielectric constant at $2143 \mathrm{MHz}$

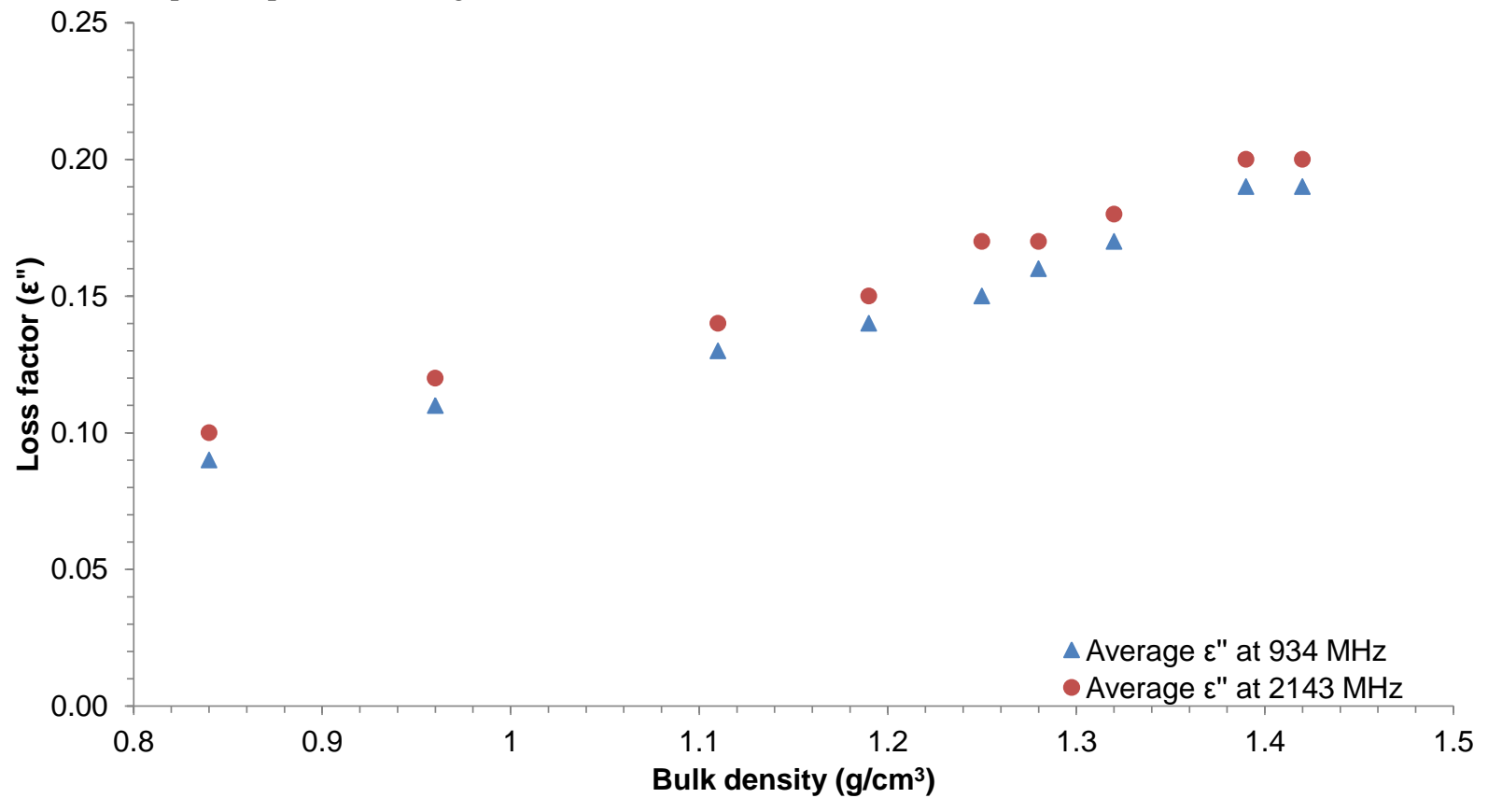

Fig. 4 Second order polynomial regression curve of the dielectric constant at 934 and $2143 \mathrm{MHz}$ of pulverised vermiculite against bulk density. The blue triangle denotes the average calculated loss factor at $934 \mathrm{MHz}$ and the red circle denotes the average loss factor at $2143 \mathrm{MHz}$ 
Table 3 Estimated solid permittivity of vermiculite from graphical extrapolations at 934 and $2143 \mathrm{MHz}$

\begin{tabular}{|c|c|c|c|c|c|c|}
\hline \multirow{2}{*}{$\begin{array}{l}\text { Frequency } \\
\quad(\mathrm{MHz})\end{array}$} & \multirow{2}{*}{$\begin{array}{l}\text { Particle } \\
\text { density } \\
\left(\mathrm{g} / \mathrm{cm}^{3}\right)\end{array}$} & \multicolumn{2}{|c|}{ Estimated $\varepsilon$ ' of solid } & \multicolumn{3}{|c|}{ Estimated $\varepsilon "$ of solid } \\
\hline & & $\begin{array}{l}\text { Polynomial } \\
\text { function }\end{array}$ & $\left(\varepsilon^{\prime}\right)^{1 / 2}=\mathrm{m} \rho+\mathrm{c}$ & $\begin{array}{l}\text { Polynomial } \\
\text { function }\end{array}$ & $\left(\varepsilon ",+\frac{\delta^{2}}{4 \alpha}\right)=\mathrm{m} \rho+\mathrm{c}$ & $\left(\varepsilon^{\prime \prime}\right)^{1 / 2}=\mathrm{m} \rho+\mathrm{c}$ \\
\hline 934 & 2.71 & 6.42 & 6.19 & 0.67 & 0.52 & 0.54 \\
\hline 2143 & 2.71 & 6.35 & 6.08 & 0.73 & 0.53 & 0.55 \\
\hline
\end{tabular}

The calculated values of dielectric constant and loss factor for the solid material are shown in Table 3. The values of the dielectric constant and loss factor of solid vermiculite have been interpolated from Figure 4 and Figure 5 at a density of $2.71 \mathrm{~g} / \mathrm{cm}^{3}$, and these are shown in Table 3. From the CM model (Equation 3) the dielectric constant was calculated to be 5.92. Graphical extrapolation estimates values of dielectric constant as 6.19 and 6.08 at 934 and $2413 \mathrm{MHz}$ respectively. The linear relationship between density and the square root of the measured dielectric constant and loss factor are shown in Figures 5 and 6. Only the data points of the measured permittivity are shown in all of the figures as the points $\rho=0$ : $\varepsilon^{\prime}=1.000649$ and $\varepsilon " '=0$ which correspond to the permittivity of air.

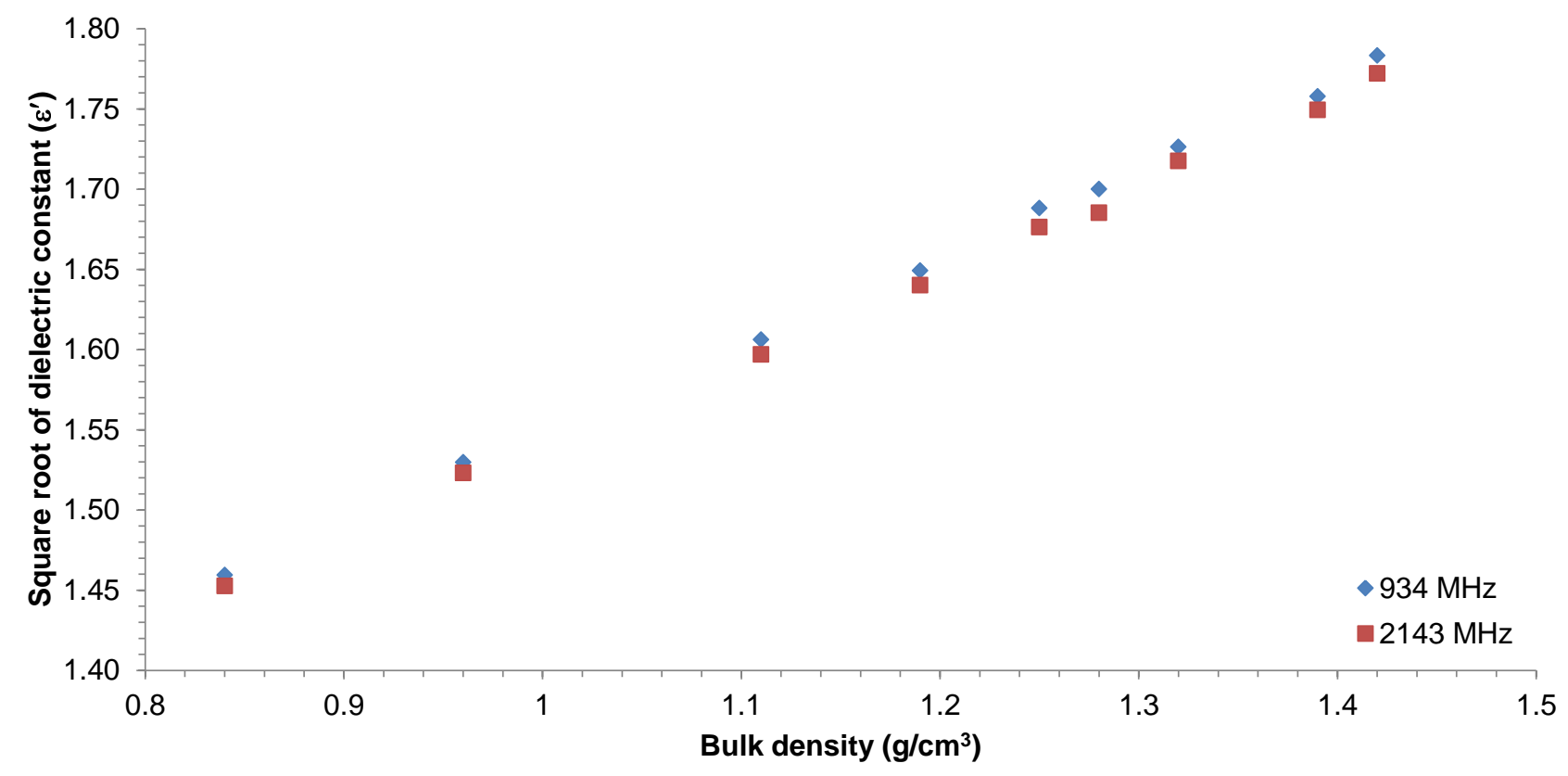

Fig. 5 Linear relationship between the square root of dielectric constant and density. The blue diamond denotes the square root of dielectric constant at $934 \mathrm{MHz}$ and the red square denotes the square root of dielectric constant at $2143 \mathrm{MHz}$ 


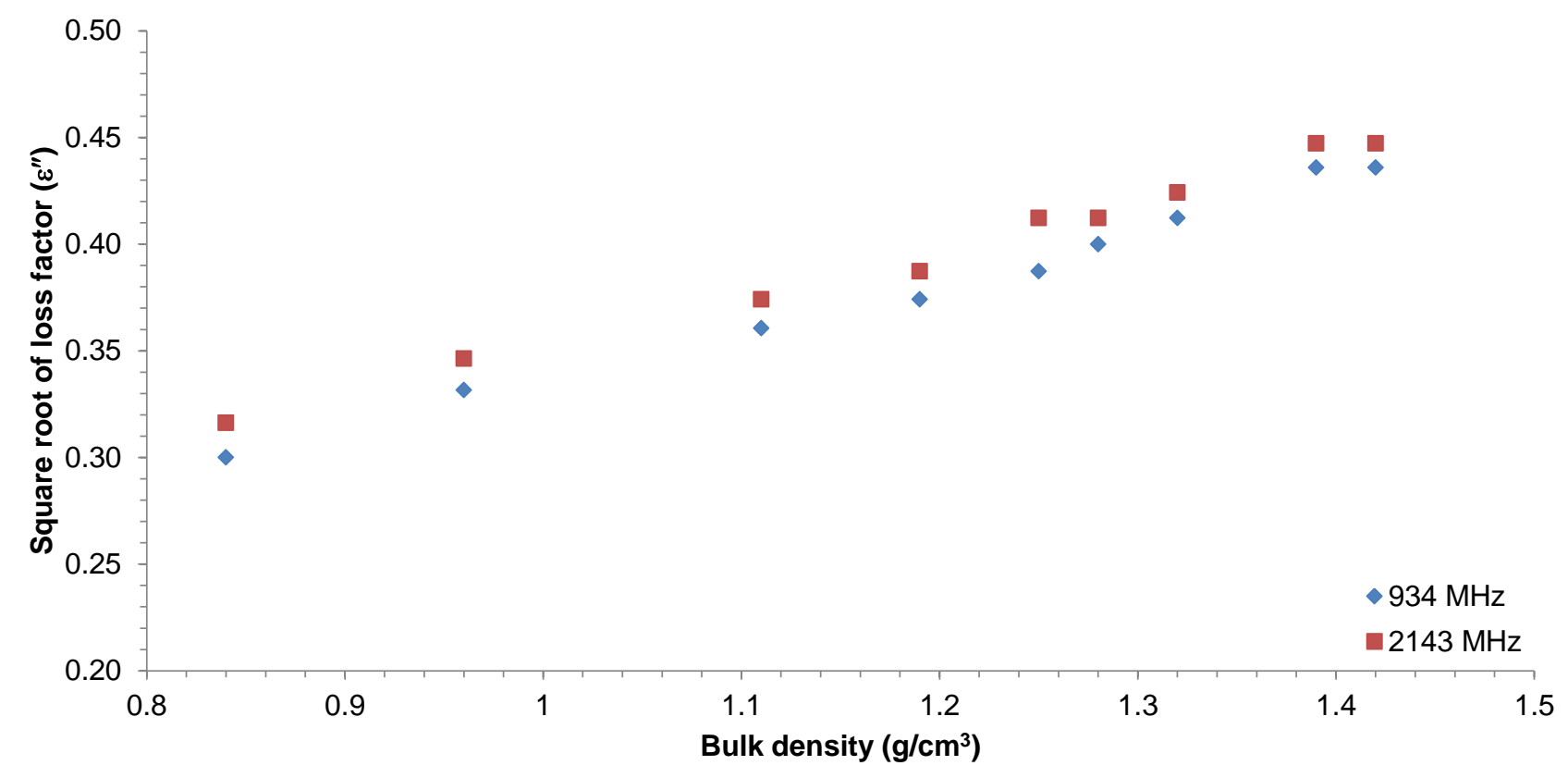

Fig. 6 Linear relationship between the square root of loss factor and density. The blue diamond denotes the square root of loss factor at $934 \mathrm{MHz}$ and the red square denotes the square root of loss factor at $2143 \mathrm{MHz}$

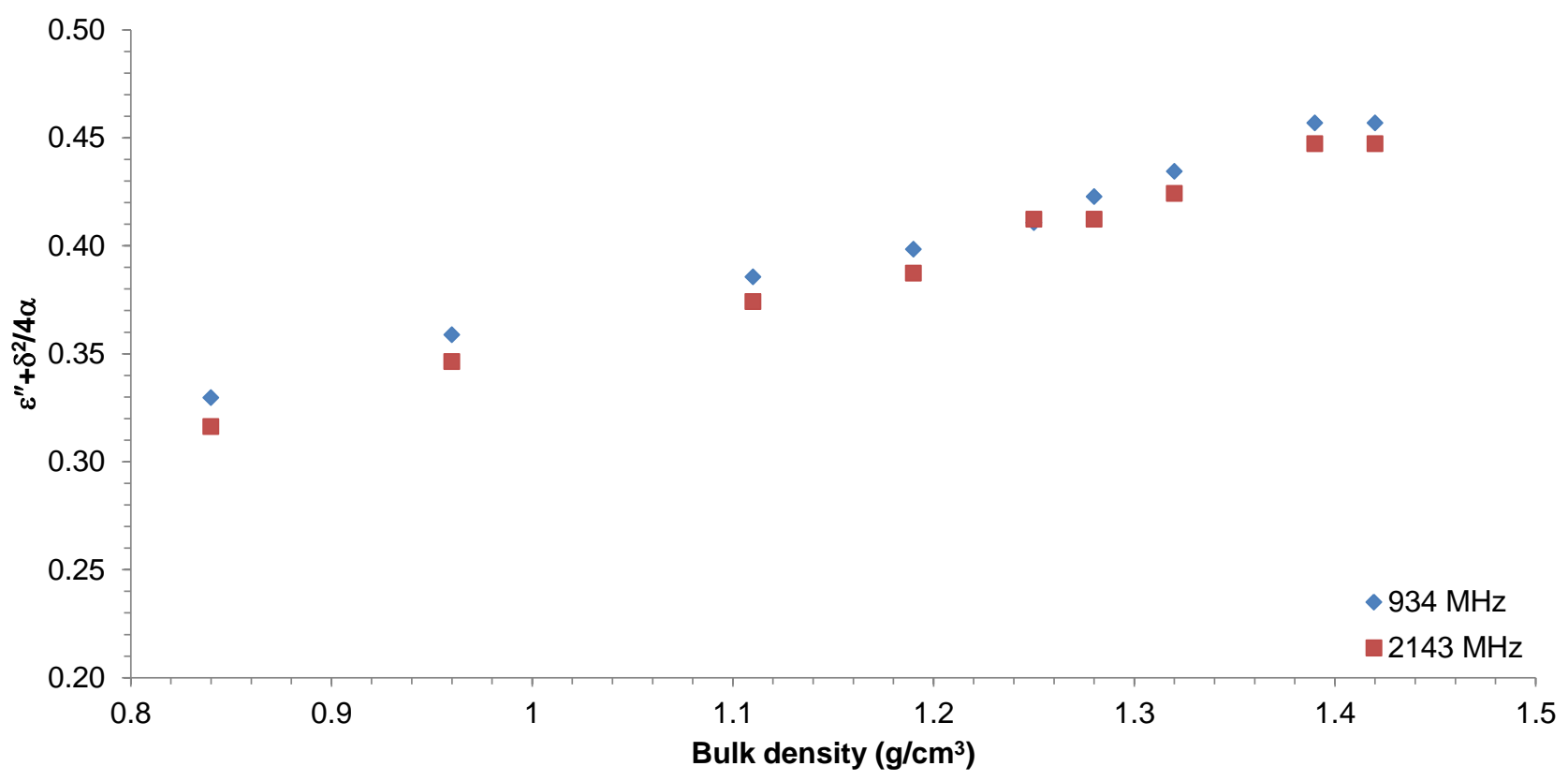

Fig. 7 Linear relationship between $\left(\varepsilon "+\frac{\delta^{2}}{4 \alpha}\right)$ and density. The blue diamond denotes $\left(\varepsilon^{\prime \prime}+\delta^{2} / 4 \alpha\right)$ at $934 \mathrm{MHz}$ and the red square denotes $\left(\varepsilon^{\prime \prime}+\delta^{2} / 4 \alpha\right)$ at $2143 \mathrm{MHz}$ 
The relationship between $\left(\varepsilon ",+\frac{\delta^{2}}{4 \alpha}\right)$ and bulk density is shown in Figure 7 . The estimated values of dielectric constant from the second order polynomial regression (Figure 3) are within less than $1 \%$ of the deviation from the measured average dielectric constant, and gives a deviation of $2-5.3 \%$ from the measured average loss factor (Figure 4). It is interesting that the loss factor interpolations from the linear relationship of the square root of the measured loss factor of the pulverised vermiculite and $\left(\varepsilon^{\prime \prime}+\frac{\delta^{2}}{4 \alpha}\right)$ against bulk density (Figure 7) respectively gives similar results at a bulk density of $2.71 \mathrm{~g} / \mathrm{cm}^{3}$. Estimation by second order polynomial for both the dielectric constant and loss factor are higher than those obtained using the linear relationship of the square-root of dielectric constant and loss factor. The regression coefficient of the square-root relationship is about $2 \%$ higher than that of the polynomial functions and produces the closet y axis intercept to 1.000649 for the dielectric constant and 0 for loss factor. This implies that the square-root relationships will give a better estimation of the dielectric constant and loss factor than the polynomial regression, and can be used with high confidence in estimating the complex permittivity of solid vermiculite from that of its pulverised form.

Table 4 shows the estimated values of the permittivity of the solid sample from the mixture rules. There is a significant variation in the estimated values using the different dielectric mixture equations due to their dependence on particle size, micro-geometry and shape of the individual particles in the mixture [4,37]. Comparison of the results obtained from the mixture rules (Table 4) and the graphical extrapolations shown in Table 3, shows that the dielectric constant and loss factor estimated by the complex refractive index, (Goldschmidt and modified Bruggeman-Hanai equation) are within 0.32-1\% of that estimated by the square-root relationship. The consistency between the complex refractive index and the square-root relationship (Equation 9) is similar to the results obtained by [4,28,37] for different plastic materials. The Landau, Lifshitz and Loyenaga equation (LLL) and Böttcher model overestimated the dielectric constant with a difference of between 11-12\% and 13-15\% from that of the value estimated by the square-root relationship while differences of between 12-20\% and 16-25\% were recorded for loss factor. This may be due to the oversimplification of the material microstructure in their derivations which are more suitable for heterogeneous materials.

The CRIM, Goldschmidt and Bruggeman-Hanai models give dielectric constant estimates comparable to those obtained from the Clausius-Mossotti equation $\left(\varepsilon^{\prime}=5.92\right)$ as discussed in Section 3.1 and these mixing rules can be used to estimate the dielectric properties of solid flakes of vermiculite. The data shown in Table 3 and 4 show that there is a close correlation within a deviation of less than $1 \%$ between the estimated dielectric constant and the loss factor of solid vermiculite from graphical interpolations of 
graphs of $\left(\varepsilon^{\prime}\right)^{1 / 2},\left(\varepsilon^{\prime \prime}\right)^{1 / 2}$ and $\left(\varepsilon^{\prime \prime}+\frac{\delta^{2}}{4 \alpha}\right)$ against bulk density at $2.71 \mathrm{~g} / \mathrm{cm}^{3}$ and the estimations from the CRIM, Goldschmidt and Bruggeman-Hanai equations. The CRIM, Goldschmidt and Bruggeman-Hanai equations have been shown to work well (deviation below $1 \%$ from the estimated value by the graphical extrapolation technique) and to be suitable for estimating dielectric properties of solid vermiculite. This information, together with the literature stating that Goldschmidt is good for multi-layered materials (deviation is below $0.5 \%$ from the estimated value using the graphical extrapolation technique), suggests that the three mixing equations may also be suitable for other multi-layered materials. The correlation of the estimated results of dielectric constant combining the $\mathrm{CM}$ equation with the results obtained from CRIM, Goldschmidt and the forms of Bruggeman-Hanai equations also indicate the possibility of calculating the dielectric properties of vermiculite from different source regions by CM, provided their molecular formulas are known with a high degree of confidence. The dielectric properties of multilayered materials such as vermiculite, validated by several techniques, gives an insight for scaling up microwave treatment of such materials. Furthermore, the work described here allows the design of an optimised cavity and choke system to operate microwave processing reliably and develops the understanding of how this process will work. Additionally, this paper gives more detailed information on the selective heating of each mineral investigated.

Table 4 Estimated solid permittivity of vermiculite from mixture rules at 934 and $2143 \mathrm{MHz}$

\begin{tabular}{|c|c|c|c|c|c|c|c|c|c|c|c|}
\hline \multirow{2}{*}{$\begin{array}{c}\text { Frequency } \\
(\mathrm{MHz})\end{array}$} & \multirow{2}{*}{$\begin{array}{c}\text { Solid density } \\
\left(\mathrm{g} / \mathrm{cm}^{3}\right) \\
\end{array}$} & \multicolumn{2}{|c|}{ CRI } & \multicolumn{2}{|c|}{ LLM } & \multicolumn{2}{|c|}{ Goldschmidt } & \multicolumn{2}{|c|}{ Böttcher } & \multicolumn{2}{|c|}{$\begin{array}{l}\text { Bruggeman- } \\
\text { Hanai }\end{array}$} \\
\hline & & $\varepsilon^{\prime}$ & $\varepsilon^{\prime \prime}$ & $\varepsilon^{\prime}$ & $\varepsilon^{\prime \prime}$ & $\varepsilon^{\prime}$ & $\varepsilon^{\prime \prime}$ & $\varepsilon^{\prime}$ & $\varepsilon^{\prime \prime}$ & $\varepsilon^{\prime}$ & $\varepsilon^{\prime \prime}$ \\
\hline 934 & 2.71 & 6.17 & 0.50 & 6.91 & 0.61 & 6.18 & 0.57 & 7.05 & 0.63 & 6.13 & 0.52 \\
\hline 2143 & 2.71 & 6.06 & 0.53 & 6.78 & 0.66 & 6.05 & 0.61 & 6.91 & 0.69 & 6.02 & 0.56 \\
\hline
\end{tabular}




\section{Conclusions}

This study has established the feasibility of the CM model to connect the dielectric constant of vermiculite to the polarizability of the molecules for such multi-layered materials. The CM model, which from first principles, is based on the ionic or atomic polarizability of the mineral composition of materials, also gives a comparable value of dielectric constant to the estimated values obtained by the CRIM, Goldschmidt and Bruggeman-Hanai equations. Experimental results show that at the selected microwave frequencies of 934 and $2143 \mathrm{MHz}$, the graphs of $\left(\varepsilon^{\prime}\right)^{1 / 2},\left(\varepsilon^{\prime \prime}\right)^{1 / 2}$ and $\left(\varepsilon^{\prime \prime}+\frac{\delta^{2}}{4 \alpha}\right)$ vary linearly with bulk density. CRIM, Goldschmidt and Bruggeman-Hanai equations were shown to be suitable for estimating the dielectric properties of solid flakes of vermiculite at its natural density of $2.71 \mathrm{~g} / \mathrm{cm}^{3}$. Graphical extrapolation of dielectric properties at $2.71 \mathrm{~g} / \mathrm{cm}^{3}$ using graphs of $\left(\varepsilon^{\prime}\right)^{1 / 2},\left(\varepsilon^{\prime \prime}\right)^{1 / 2}$ and $\left(\varepsilon^{\prime \prime}+\frac{\delta^{2}}{4 \alpha}\right)$ against density also give reasonably close values within $0.1-1 \%$ deviations to the estimated values obtained from CRIM, Goldschmidt and Bruggeman-Hanai equations. The Goldschmidt equation, earlier stated in the literature to be suitable for estimating dielectric properties of multi-layered materials, CRIM equation and a modified form of the Bruggeman-Hanai equation have been shown to provide good estimates for the dielectric properties of solid vermiculite. These mixing rules may also be suitable for estimating the dielectric properties of other multi-layered phyllosilicate materials and therefore the use of these mixture equations to accurately estimate the dielectric properties of multi-layered materials may be possible. If so it would then become possible to design more efficient microwave systems. 


\section{References}

1. Gallego Carrera D, Mack A (2010) Sustainability assessment of energy technologies via social indicators: Results of a survey among European energy experts. Energy Policy 38 (2):1030-1039

2. Robinson J, Kingman S, Irvine D, Licence P, Smith A, Dimitrakis G, Obermayer D, Kappe CO (2010) Understanding microwave heating effects in single mode type cavities-theory and experiment. Physical Chemistry Chemical Physics 12 (18):4750-4758

3. Tuhkala M, Juuti J, Jantunen $H$ (2013) Method to characterize dielectric properties of powdery substances. Journal of Applied Physics 114 (1):014108-014108-014108

4. Nelson SO Estimation of permittivities of solids from measurements on pulverised or granular materials. In: Kong JA (ed) Progress in Electromagnetics Research, New York, 1992. Elsevier Science and Technology, pp Chapter 6 , 231-271

5. Metaxas A.C; Meredith R.J (1983) Industrial microwave heating. 1st Edition edn. The Instritute of Engineering and Technology, London

6. Wang M, Pan N (2008) Predictions of effective physical properties of complex multiphase materials. Materials Science and Engineering: R: Reports 63 (1):1-30

7. Olaosebikan F, Christopher D, Georgios D, Samuel K (2012) Continuous energy efficient exfoliation of vermiculite through microwave heating. International Journal of Mineral Processing 114-117:69-79. doi:http://dx.doi.org/10.1016/i.minpro.2012.10.003

8. Kingman S, Vorster W, Rowson N (2000) The influence of mineralogy on microwave assisted grinding. Minerals Engineering 13 (3):313-327

9. Jones D, Lelyveld T, Mavrofidis S, Kingman S, Miles N (2002) Microwave heating applications in environmental engineering-a review. Resources, conservation and recycling 34 (2):75-90

10. Vorster W, Rowson N, Kingman S (2001) The effect of microwave radiation upon the processing of Neves Corvo copper ore. International Journal of Mineral Processing 63 (1):29-44

11. Ballantyne G, Holtham P (2010) Application of dielectrophoresis for the separation of minerals. Minerals Engineering 23 (4):350-358

12. Foster MD (1960) Interpretation of the composition of trioctahedral micas. US Government Printing Office,

13. Newman A, Brown G (1966) Chemical changes during the alteration of micas. Clay Miner 6 (29):310

14. El Mouzdahir Y, Elmchaouri A, Mahboub R, Gil A, Korili SA (2009) Synthesis of nano-layered vermiculite of low density by thermal treatment. Powder Technology 189 (1):2-5

15. Bain D, Smith B (1994) Chemical analysis. In: Clay mineralogy: Spectroscopic and chemical determinative methods. Springer, pp 300-332

16. Hippel ARV (1954) Dielectrics and waves. 3rd edn. John Wiley and Sons, Massachusetts

17. Sucher M, Fox J, Wind M (1963) Handbook of microwave measurements, vol 2. Polytechnic Press of the Polytechnic Institute of Brooklyn,

18. Venkatesh MS, Raghavan GVS (2005) An overview of dielectric properties measuring techniques. Canadian Biosystems Engineering 47 (7):15-30

19. Baker-Jarvis J, Geyer R, Grosvenor J, Janezic M, Jones C, Riddle B, Weil C, Krupka J (1998) Dielectric characterization of low-loss materials a comparison of techniques. IEEE Transactions on Dielectric and Electrical Insulation 5 (4)

20. Anderson JC (1964) Dielectrics. Chapman and Hall London,

21. Smith AD, Lester EH, Thurecht KJ, Kingman SW, El Harfi J, Dimitrakis G, Robinson JP, Irvine DJ (2010) Temperature Dependence of the Dielectric Properties of 2, 2'-Azobis (2-methyl-butyronitrile)(AMBN). Industrial \& Engineering Chemistry Research 49 (6):3011-3014

22. Adams F, De Jong M, Hutcheon R (1992) Sample shape correction factors for cavity perturbation measurements. Journal of Microwave Power and Electromagnetic Energy 27 (3):131-135 
23. Clarke B, Gregory A, Cannell D, Patrick M, Wylie S, Youngs I, Hill G (2003) A Guide to the Characterisation of Dielectric Materials at Rf and Microwave Frequencies. Institute of Measurement and Control/National Physical Laboratory.

24. Hilhorst M, Dirksen C, Kampers F, Feddes R (2000) New dielectric mixture equation for porous materials based on depolarization factors. Soil Science Society of America Journal 64 (5):1581-1587

25. Doyle WT (1978) The Clausius-Mossotti problem for cubic arrays of spheres. Journal of Applied Physics 49 (2):795-797. doi:doi:http://dx.doi.org/10.1063/1.324659

26. Shannon R.D (1993) Dielectric polarizabilities of ions in oxides and fluorides. Journal of Applied Physics 73 (1):348-366. doi:10.1063/1.353856

27. Robinson DA (2004) Calculation of the dielectric properties of temperate and tropical soil minerals from ion polarizabilities using the clausius mosotti equation. Soil Science Society American Journal 68:1780-1785

28. Nelson SO (1994) Measurement of microwave dielectric properties of particulate materials. Journal of Food Engineering 21 (3):365-384

29. Sheen J (2009) Measurements of microwave dielectric properties by an amended cavity perturbation technique. Measurement 42 (1):57-61

30. Sihvola A (2000) Electromagnetic mixing formulas and applications, vol 1. IEE Electromagnetic Wave 1 edn. The institution of Electrical Engineers, London

31. Sihvola A (2000) Mixing rules with complex dielectric coefficients. Subsurface Sensing Technologies and Applications 1 (4):393-415

32. Reynolds J, Hough J (1957) Formulae for dielectric constant of mixtures. Proceedings of the Physical Society Section B $70(8): 769$

33. Knoll MD (1996) A petrophysical basis for ground penetrating radar and very early time electromagnetics: Electrical properties of sand-clay mixtures.

34. Chang C.S (1988) Measuring density and porosity of grains kernels using a gas pycnometer. American Society of Cereals Chemists 65 (1):13-12

35. Kent M (1977) Complex permittivity of fish meal: a general discussion of temperature, density and moisture dependence

Journal of Microwave Power 12:341-345

36. Klein A (1981) Microwave determination of moisture in coal-comparison of attenuation and phase measurement. Journal of Microwave Power and Electromagnetic Energy 16 (3 \& 4):289-304

37. Nelson S.O, You T.S (1989) Relationships between microwave permittivities of solids and pulverised plastics. Journal of Physics D: Applied Physics 23:346-353

38. Metaxas A, Meredith R (1988) Industrial Microwave Heating, Peter Peregrinus Ltd., London(1988).

39. Kent M (1977) Complex permitivity of fish-meal-general discussion of temperature, desnity and moisture dependance. Journal of microwave power and electromagnetic energy 12 (4):341-345 